\title{
On Representing Graphs by Touching Cuboids
}

\author{
David Bremner ${ }^{1}$, William Evans ${ }^{2}$, Fabrizio Frati ${ }^{3}$, Laurie Heyer ${ }^{4}$, \\ Stephen G. Kobourov ${ }^{5}$, William J. Lenhart ${ }^{6}$, Giuseppe Liotta ${ }^{7}$, \\ David Rappaport ${ }^{8}$, and Sue H. Whitesides ${ }^{9}$ \\ ${ }^{1}$ Faculty of Computer Science, University of New Brunswick \\ 2 Department of Computer Science, University of British Columbia \\ 3 School of Information Technologies, The University of Sydney \\ ${ }^{4}$ Department of Mathematics, Davidson College \\ 5 Department of Computer Science, University of Arizona \\ 6 Department of Computer Science, Williams College \\ 7 Department of Computer Science, University of Perugia \\ 8 School of Computing, Queens University \\ ${ }^{9}$ Department of Computer Science, University of Victoria
}

\begin{abstract}
We consider contact representations of graphs where vertices are represented by cuboids, i.e. interior-disjoint axis-aligned boxes in 3D space. Edges are represented by a proper contact between the cuboids representing their endvertices. Two cuboids make a proper contact if they intersect and their intersection is a non-zero area rectangle contained in the boundary of both. We study representations where all cuboids are unit cubes, where they are cubes of different sizes, and where they are axis-aligned 3D boxes. We prove that it is NP-complete to decide whether a graph admits a proper contact representation by unit cubes. We also describe algorithms that compute proper contact representations of varying size cubes for relevant graph families. Finally, we give two new simple proofs of a theorem by Thomassen stating that all planar graphs have a proper contact representation by touching cuboids.
\end{abstract}

\section{Introduction}

There is a large body of research about representing planar graphs as contact graphs, i.e. vertices are represented by geometrical objects and edges correspond to pairs of objects touching in some specified fashion (see, e.g., [1, 3, 5, 8, 9, 13, 15, 16]). Typical classes of objects might be curves, line segments, or polygons. An early result is Koebe's 1936 theorem [17] stating that all planar graphs can be represented by touching disks.

Here we consider contact representations of graphs with vertices represented by cuboids, i.e., interior-disjoint axis-aligned boxes in 3D space. Graph edges are represented by contacts between cuboids, i.e., there is an edge if and only if two cuboids touch, where two cuboids touch if they intersect and their intersection is contained in the boundary of both. In particular, we are interested in the class of planar graphs that can be represented by proper contact of cuboids, where proper contacts must have non-zero area. Cubes are cuboids such that all sides have the same length.

\subsection{Related Work}

Cuboids become rectangles in the $2 \mathrm{D}$ version of this problem, and such a representation is known as a rectangular layout of the input planar graph. There are several 
(independent) characterizations of the class of planar graphs that allows such rectangular layouts [18, 19, 23]. A historical overview and a summary of the state of the art in the rectangle contact graphs literature can be found in Buchsbaum et al. [6] and Felsner [11].

For 3D, Thomassen [22] shows that any planar graph has a proper contact representation by cuboids. Felsner and Francis [12] prove that any planar graph has a non-proper contact representation by cubes, where a valid contact between cubes that represent adjacent vertices may have zero area. This raises the question of whether a proper contact representation by cubes is possible for all planar graphs.

\subsection{Our Contributions}

- We show that any graph with $n$ vertices that admits a proper contact representation by unit cubes has at most $7 n-\Omega\left(n^{\frac{2}{3}}\right)$ edges, and this bound is tight. We give families of planar graphs that do and do not admit proper contact representation by unit cubes (Section 2).

- We prove that the problem of deciding whether a graph admits a proper contact representation by unit cubes is NP-complete (Section 3).

- We give classes of (non-planar) graphs that have no proper contact representation even if cubes are of varying sizes. We show that varying size cubes can represent larger graph classes, such as partial planar 3-trees, than unit cubes (Section 4).

- We describe two new proofs of Thomassen's result [22]. The first one uses the canonical ordering of de Fraysseix, Pach and Pollack [14]; the second one uses Schnyder's realizers [21] (Section 5).

\section{Properties of Unit Cube Representations}

Here we establish basic combinatorial properties of graphs with proper contact representations by unit cubes. First we study the classical Turán-type problem of finding an upper bound on the number of edges as a function of the number of vertices. We then give graphs that do and do not have a proper contact representation by unit cubes.

We say two cubes make a vertex contact if each cube has a vertex $v$ lying in the interior of a face of the other cube; then $v$ participates in a vertex contact. Two cubes make an edge contact if each cube has an edge $e$ lying in the face of the other cube; then the endpoints of e participate in an edge contact. Two cubes make a face contact if each cube has a face $f$ coincident with a face of the other cube; then the vertices of $f$ participate in a face contact.

Theorem 1. The maximum possible vertex degree of a graph that admits a proper contact representation by unit cubes is 14 .

Proof: Consider any proper contact representation by unit cubes of a graph $G$ containing a vertex of degree 14. Let $C$ be the cube representing a degree-14 vertex in the representation of $G$. We label each vertex $v$ of $C$ with the multi-set of contact types it participates in: $\mathrm{v}$, e, and $\mathrm{f}$ for vertex, edge, and face contact, respectively. If $v$ participates in a vertex contact, it cannot participate in another vertex contact, and it can 
participate in at most one edge contact and one face contact, or in two face contacts. If $v$ does not participate in a vertex contact and it does participate in an edge contact, it can participate in at most one more edge contact and one face contact, or in two face contacts. If $v$ only participates in face contacts, it can participate in at most three of them. Thus a valid label of $v$ is a subset of $\{v, e, f\},\{v, f, f\},\{e, e, f\},\{e, f, f\}$, or $\{f, f, f\}$. Every e label at a vertex $v$ of $C$ implies another e label at a vertex of $C$ adjacent to $v$, caused by the same cube touching $C$. Thus, the total number of e labels at vertices of $C$ is twice the number of adjacent cubes contributing to those labels, i.e. each e label contributes $\frac{1}{2}$ to the number of cubes adjacent to $C$. Every f label at a vertex $v$ of $C$ implies three other $\mathrm{f}$ labels at different vertices of $C$ caused by the same cube touching $C$, so each $\mathrm{f}$ label contributes $\frac{1}{4}$ to the degree of $C$. Every v label contributes 1 to the degree of $C$. The total contribution to the degree of $C$ given by a valid vertex label is at most 1.75 , as provided by $\{\mathrm{v}, \mathrm{e}, \mathrm{f}\}$. As $C$ has 8 vertices, its degree is at most 14 .

We are now ready to establish an upper bound on the number of edges in a graph that admits a proper contact representation by unit cubes.

Theorem 2. If a graph $G$ with $n$ vertices has a proper contact representation by unit cubes then it has at most $7 n-\Omega\left(n^{2 / 3}\right)$ edges, which is asymptotically tight.

Proof: Let $\Gamma$ be any unit cube representation of $G$. From the proof of Theorem 1, a cube achieves proper contact with the maximum of 14 other cubes only if every vertex of the cube is of type $\{v, e, f\}$ (i.e. involved a vertex, an edge, and a face contact). No $\{\mathrm{v}, \mathrm{e}, \mathrm{f}\}$ cube vertex is visible from infinity, so to establish the bound it suffices to show $\Omega\left(n^{2 / 3}\right)$ cubes in $\Gamma$ have vertices visible from infinity. The maximum area axis parallel view of $\Gamma$ has area at least $n^{2 / 3}$. Since no face of the corresponding planar map has more than unit area, there are $\Omega\left(n^{2 / 3}\right)$ cube vertices visible from infinity. Since each cube contributes at most 4 such vertices, the bound follows.

To prove that this upper bound is tight, we pack $n=k^{3}$ ( $k$ odd) unit cubes in an axis-aligned bounding box $B$ of volume $n$ and side length $k$. For $i=1, \ldots, k$, let $L_{i}$ be the set of $k^{2}$ cubes with $z$-coordinate in $[i, i+1]$. For every even $i$, shift $L_{i}$ by $(\epsilon, \epsilon, 0)$ (with $0<\epsilon<1$ ) so that each cube of $L_{i}$, except for those originally on the border of $B$, touches 4 cubes of $L_{i-1}$ and $L_{i+1}$. For $j=1, \ldots, k$, let $R_{i j}$ be the row of $k$ cubes in $L_{i}$ with $y$-coordinate in $[j, j+1]$. For every even $i$ and $j$, shift $R_{i, j}$ by $(\epsilon, 0,0)$ so that each cube of $R_{i, j}$, except for those originally on the border of $B$, touches two cubes of $R_{i, j-1}$ and $R_{i, j+1}$. Now every cube, except for the $O\left(n^{2 / 3}\right)$ cubes originally on the border of $B$, has degree 14 .

As the next lemma shows, volume constraints prevent some graphs with maximum vertex degree less than 14 from having a proper contact representation by unit cubes.

Lemma 1. Every complete binary tree whose height is larger than or equal to 14 does not admit a proper contact representation by unit cubes.

Proof: Let $T$ be a complete binary tree with $n=2^{h+1}-1$ vertices and height $h$. The diameter of $T$ is $2 h$. Since $T$ is connected, a proper contact representation of $T$ by unit cubes has a bounding box with side lengths at most $2 h+1$ and thus volume at most $(2 h+1)^{3}$. As the volume must be at least $n$, a contradiction results for $h \geq 14$. 

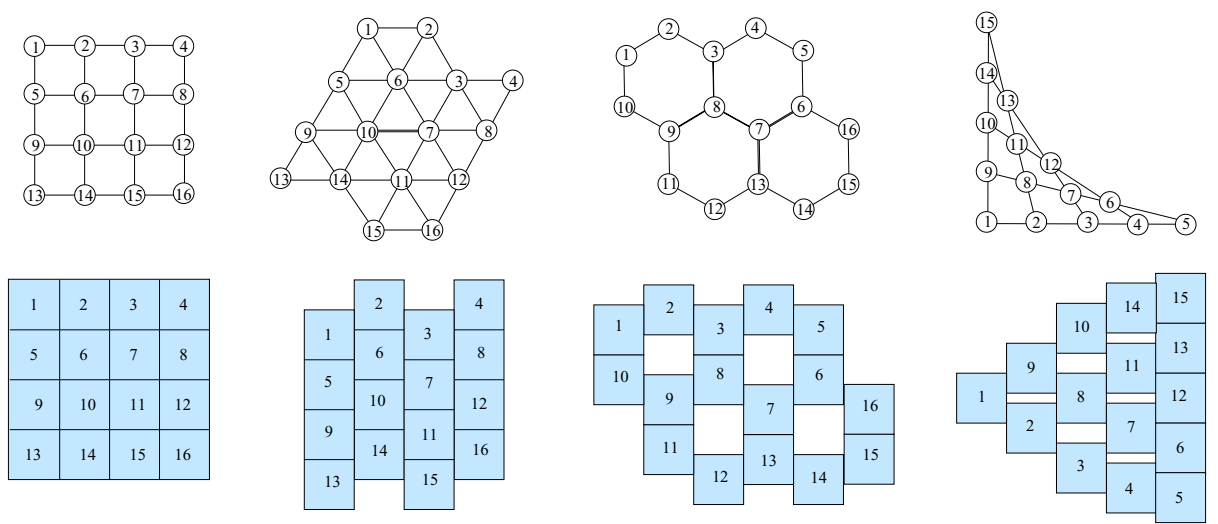

Fig. 1. Regular tiling patterns with unit cubes

By the argument above, any graph containing a vertex $v$ such that the number of vertices with graph-theoretic distance at most $d$ from $v$ is greater than $(2 d+1)^{3}$ does not have a proper contact representation by unit cubes. The next theorem gives classes of planar graphs that $d o$ have a proper contact representation by unit cubes.

Theorem 3. Triangular grid, square grid, hexagonal grid, and parabolic grid graphs have a proper contact representation by unit cubes.

Proof Sketch: See Fig. 1, which gives regular tiling patterns (infinitely extendable) for the claimed grid graphs.

\section{Complexity of the Unit Cube Representability Problem}

The hardness part of our proot that it is NP-complete to determine whether a graph has a proper contact representation by unit cubes goes by polynomial-time reduction from NAE 3-SAT, defined as follows: Given a formula $F$ with $m$ clauses and $n$ variables, is there a truth assignment for the variables in $F$ such that each clause contains at least one satisfied literal and at least one unsatisfied literal?

Section 3.1 gives a design for a "logic engine" (see [10]), which is essentially a graph constructed from a formula $F$, such that if $F$ is satisfiable, then the graph admits a proper contact representation by unit cubes. In particular, the graph is to be the proper contact graph of a certain assembly of unit cubes. We define the graph implicitly by describing the logic engine as this assembly of cubes. There are options for the placement of some of the subassemblies that result in the same underlying contact graph. If $F$ is satisfiable, then combinations of these options can be chosen so that there are no collisions between subassemblies, and so that the assembly is a proper contact representation of the graph. Section 3.2 uses the results of Section 2 to give tools for proving that the graph admits a proper contact representation by unit cubes only if $F$ is satisfiable. Section 3.3 completes the proof sketch.

\footnotetext{
${ }^{1}$ An alternate proof based on techniques from Breu's $\mathrm{PhD}$ thesis [5] also seems possible.
} 


\subsection{Logic Engine Design}

The logic engine assembly of unit cubes is comprised of subassemblies called blocks, where a block is an $a \times a \times a$ cube of unit cubes. A schematic cross-section of the logic engine for an $m=n=3$ instance is shown in Fig. 2. Depending on its placement in the assembly, we say a block is a pole block, a spacer block, a link block, or a flag block. The entire assembly is contained by a shell, which is an assembly of unit cubes in the form of a hollow rectangular box with walls that are 2 unit cubes thick. Inside the shell is the shaft, which is a subassembly consisting of a sequence of pole and spacer blocks, adjoined by unit cubes and attached to the side walls of the shell. The pole blocks support pole assemblies, which consist of link blocks adjoined to unit cubes. Each pole is associated with a variable; half the pole is associated with the uncomplemented variable and the other with its complement. The positions of the halves can be interchanged by rotating the pole block $180^{\mathrm{deg}}$ with respect to its adjoining unit cubes on the shaft, thus turning the pole about the shaft. Flag blocks may be attached to link blocks along the poles, forming a rectanguloid double block of dimensions $2 a \times a \times a$. A double block may be rotated relative to its adjacent unit cubes, thus turning it about the pole.

For an instance of NAE 3-SAT with $m$ clauses and $n$ variables, there are $n$ poles, each containing $m$ link blocks in each half-pole. A flag block is attached to the $i^{t h}$ link block from the $j^{\text {th }}$ pole block, on the uncomplemented side, if and only if the $i^{\text {th }}$ clause fails to contain the $j^{\text {th }}$ variable (uncomplemented); similarly, a flag block is attached to the $i^{\text {th }}$ link block on the complemented side of the $j^{\text {th }}$ pole if and only if the $i^{\text {th }}$ clause does not contain the $j^{\text {th }}$ variable in complemented form. Along the poles and the shaft, spacer blocks separate link and pole blocks.

The value of $a$ is chosen odd so that each block face has a unit cube face at its center. Each unit cube depicted in Fig. 2 makes face contact on two sides with a unit cube in the center of the face of a neighboring block (or shell); this allows poles and flagged link blocks to "turn" independently. Unit cubes are shown larger than scale.

A variable is regarded as "true" if the uncomplemented part of the pole is positioned above the shaft. The flags can be positioned so that they do not intersect other flags or the shell if there is at least one unflagged block in each row of blocks above and below the shaft, in which case all the remaining flags in the row can be rotated to point to the unflagged block. This corresponds to having at least one true literal and at least one false literal in each clause. Fig. 2 encodes a "yes" instance of $\left(x_{1} \vee x_{2} \vee \overline{x_{3}}\right) \wedge\left(x_{1} \vee\right.$ $\left.x_{2} \vee x_{3}\right) \wedge\left(\overline{x_{1}} \vee x_{2} \vee x_{3}\right)$, where $x_{1}=T, x_{2}=F$, and $x_{3}=T$.

\subsection{Making the Blocks and Shell Rigid}

In order to establish the equivalence between a NAE 3-SAT instance and an instance of the graph realizability by touching unit cubes, we need to ensure the "rigidity" of the shell and of the blocks. This is accomplished by the introduction of additional graph structure, which is described in the following.

A cube graph is the skeleton of a cube, i.e. a matched pair of chordless 4-cycles. The contact graphs corresponding to the shell and to the blocks are composed of a set of cube graphs. The rigidity of such parts of the contact graph will ensure that the walls of the shell and the blocks have thickness 2. It is not hard to show that if the 8 vertices in 


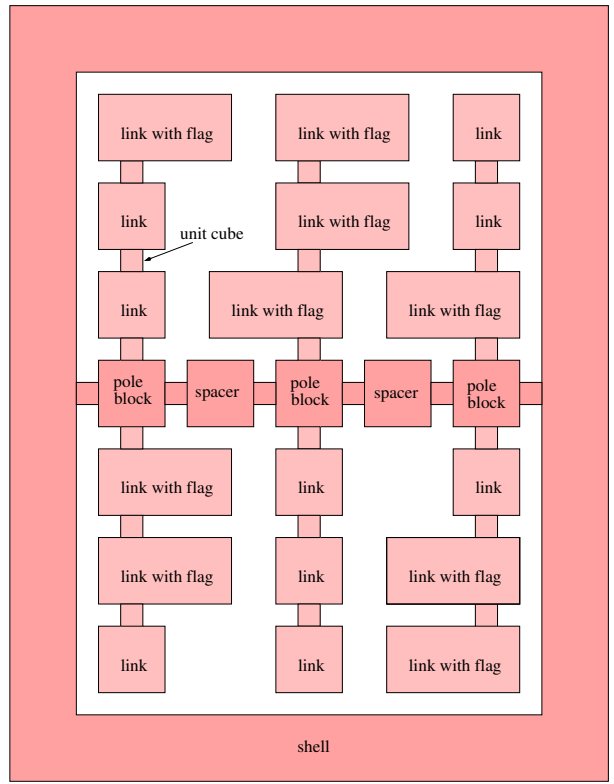

Fig. 2. Schematic cross-section of the logic engine (shaft and shell are darkly shaded)

any cube subgraph in a block or shell must be realized tightly, i.e. by unit cubes within a $2 \times 2 \times 2$ bounding box, then the entire block or shell must be represented in the desired aligned and rigid way. Our proof defines and uses crystal lattice graphs, inspired by crystallography (see, e.g., [4]). In order to force the representation of a cube graph to be tight (hence forcing the rigidity of the shell or block to which the cube graph belongs), we attach the cube graph to a crystallizer graph, which in turn contains a seed graph. These graphs are described below.

It follows from the proof of Theorem 1 that the only way a cube $C$ can represent a vertex of degree 14 in a unit cube representation of a graph is if every vertex of $C$ has label $\{\mathrm{v}, \mathrm{e}, \mathrm{f}\}$. That implies that two opposite faces of $C$ have face contact. Label the vertices of $C$ from 0 to 7 so that vertex $i$ and vertex $j$ lie on the same cube edge if and only if their binary representations differ in a single bit and the two faces of $C$ making face contacts are $\{0,1,2,3\}$ and $\{4,5,6,7\}$ (see Fig. 3(c)). None of the edges connecting these two faces can be part of an edge contact, as otherwise the endpoints of the edge could not be part of a vertex contact. Since no vertex participates in two edge contacts, the four edges of $C$ that make edge contact cannot share a common vertex. Assume without loss that two of the four edge contacts touch $\{4,6\}$ and $\{5,7\}$. The other two edge contacts might touch $\{0,2\}$ and $\{1,3\}$ (see Fig. 3 (a)) or they might touch $\{0,1\}$ and $\{2,3\}$ (see Fig. 3 (b)). In the first case, we say that the realization is of type $(a)$; in such a realization, four of the eight cubes making vertex contact with $C$ touch face $\{2,3,6,7\}$ (the top face of $C$ ) and four touch face $\{0,1,4,5\}$ (the bottom face). In the second case, we say that the realization is of type $(b)$; in such a realization, each pair of the eight cubes making vertex contact with $C$ touches a different face of $C$. 


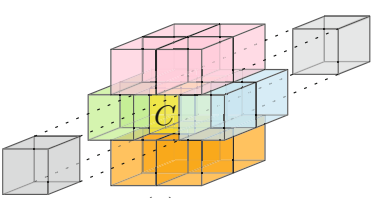

(a)

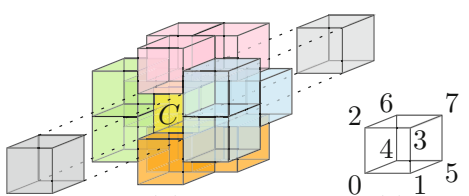

(b)

(c)

Fig. 3. (a)-(b) The two types of realizations of a degree-14 cube $C$. (c) The labels on the vertices of cube $C$.

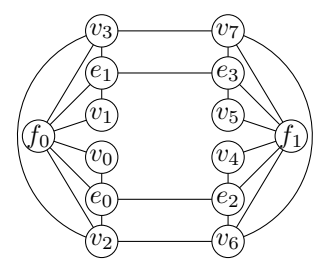

Fig. 4. Graph $G_{0}-\{c\}$ used in the proof of Lemma2

Let $G_{0}$ be a graph with 15 vertices, one of which has degree 14 (denote by $c$ such a vertex), such that the neighbors of $c$ induce the graph in Fig. 4. The next lemma shows that $G_{0}$ can be realized by touching unit cubes only if the realization of $c$ is of type (a). We say that a set of cubes is extremal in a representation if they all have a face lying on the same plane, which separates them from the rest of the cubes in the representation. We say that 4 cubes form a tight 4-cycle if their union is a box of size $2 \times 2 \times 1$.

Lemma 2. Any realization of $G_{0}$ by touching unit cubes is of type (a). Moreover, $G_{0}$ can be realized by touching unit cubes only if the four vertices $v_{2}, v_{3}, v_{6}$, and $v_{7}$ are extremal in the representation of $G_{0}$.

Proof: Consider any realization of $G_{0}$ by touching unit cubes. Let $C$ be the cube that represents vertex $c$. Vertices $f_{0}$ and $f_{1}$ have degree 6 in $G_{0}-\{c\}$ and thus must be represented by the cubes that make face contact with $C$ since these are the cubes that properly contact that many cubes. Vertices $v_{0}, v_{1}, v_{4}$, and $v_{5}$ have degree 2 in $G_{0}-\{c\}$ and thus must be represented by cubes that make vertex contact with $C$ since each cube that makes an edge contact with $C$ is adjacent to at least three cubes different from $C$ in any representation of $G_{0}$ by touching unit cubes. Vertices $e_{0}, e_{1}, e_{2}$, and $e_{3}$ must be represented by the four cubes in edge contact with $C$ since the cubes representing vertices $v_{0}, v_{1}, v_{4}$, and $v_{5}$ must each contact one edge contact cube and one face contact cube. Since $e_{0}$ and $e_{2}$ are adjacent, the representation must be of type (a) (Fig. 3), since type (b) does not allow cubes in edge contact with $C$ to touch. Finally, since vertices $\left\{v_{2}, v_{3}, v_{7}, v_{6}\right\}$ form a cycle and are represented by cubes that make vertex contact with $C$ (the only remaining kind of contacts with $C$ ), they must be represented by cubes that touch the same face of $C$ and are extremal.

We say that the chordless 4 -cycle $(2,3,6,7)$ of $G_{0}$, which by Lemma 2 must be represented by an extremal 4-cycle of cubes, lies in contact with the top face of $C$. 


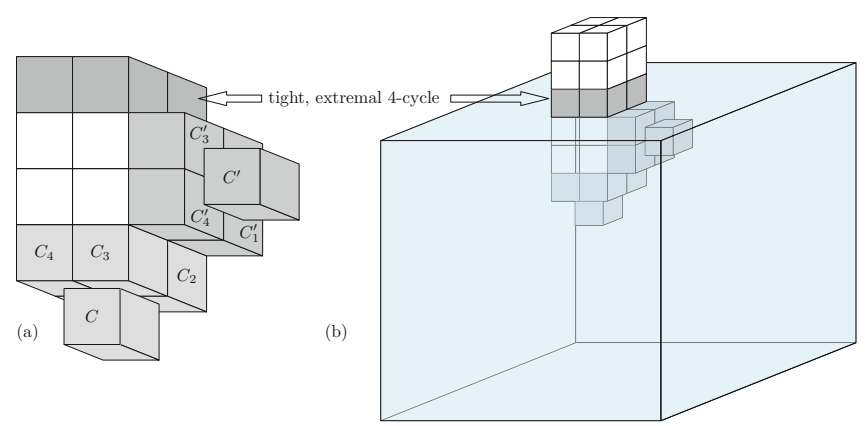

Fig. 5. (a) A portion of the seed graph. $C$ and $C^{\prime}$ represent vertices of degree 14, having $\left(C_{1}, C_{2}, C_{3}, C_{4}\right)$ and $\left(C_{1}^{\prime}, C_{2}^{\prime}, C_{3}^{\prime}, C_{4}^{\prime}\right)$ as "top" cycles of the neighbors. The remaining neighbors of $C$ and $C^{\prime}$ are not shown. (b) The crystallizer graph, with a $10 \times 10 \times 10$ box enclosing all but the tight, extremal 4-cycle of its seed graph. Only a portion of the seed graph is shown.

To create the seed graph, we take the representations of two copies of the graph $G_{0}$, as described above, and attach the top 4-cycle of each representation to a tight representation of a central cube graph, as shown in Fig. 5. The two top 4-cycles must be represented tightly, with no hole as in Fig. 5(b), because the face planes of the top layers align the cubes. Finally, we match an additional chordless 4-cycle, the extremal 4-cycle, to a chordless 4-cycle in the central cube graph, as shown in the upper part of Fig. 5(a), to obtain the seed graph $G_{1}$. The crystallizer graph is a cube graph with the vertices of one of its chordless 4-cycles matched to the vertices of the tight, extremal chordless 4-cycle of the seed graph; see Fig. 5(b). This cube graph is always tight in any crystallizer graph representation, and hence forces the rigid alignment of the representations of the crystal lattice graphs, as well as the rigidity of the graphs for the blocks and the shell when it is identified with one of their cube subgraphs.

\subsection{Recognizing Proper Contact Graphs of Unit Cubes Is NP-Complete}

To prove membership in NP, it suffices to prove that if a representation exists, then one with a polynomially bounded description exists; this can be done by a technique similar to that of the proof of Theorem 3.2 of Czyzowicz et al. [7].

Any instance of NAE-3SAT can be transformed in polynomial time to a logic engine graph made of crystal lattice graphs for the shell, link, flag, pole, and spacer blocks together with the crystallizer graphs that make these parts rigid, together with the vertices whose representations allow the poles and links to turn independently (i.e. to be spatially positioned in alternative ways). If the NAE-3SAT instance can be satisfied, then the logic engine graph can be properly realized in at least one way (see Section 3.1). On the other hand, if the logic engine graph can be represented, then its crystallizer subgraphs force the parts to be drawn rigidly and aligned; the shell forces the poles, links and flags into a flattened configuration, such that an unflagged position must appear in each row above and below the shaft to avoid intersection of unit cubes. Thus each clause has at least one true and one false literal. 


\section{Cubes of Varying Size}

Motivated by the result of the previous section, we consider graphs that admit a proper contact representation by cubes of varying size. We start by establishing an upper bound on the number of edges that such graphs can have. The proof follows from Theorem 1 .

Theorem 4. If a graph $G$ with $n$ vertices has a proper contact representation by cubes then it has at most $14 n$ edges.

We now show that a fairly general family of planar graphs, namely partial planar 3trees, has a proper contact representation by cubes. A partial planar 3-tree is a planar graph with tree-width at most 3 . Outerplanar graphs and series-parallel graphs are partial planar 3-trees. Partial planar 3-trees with $n$ vertices can have vertices of degree $\Omega(n)$; hence, by Theorem 1 they do not have a proper contact representation by unit cubes. The following theorem can be proved by combining the result by Felsner and Francis [12] with a result by Badent et al. [2]. In the first paper, the authors show that starting from a representation as a contact graph of equilateral triangles, where no two triangles touch corner-to-corner, a representation by touching cubes can be constructed. In the second paper, the authors show that partial planar 3-trees have a representation as contacts of equilateral triangles, such that no two triangles touch corner-to-corner.

Theorem 5. Every partial planar 3-tree has a proper contact representation by cubes.

\section{Proper Contact Representations by Cuboids}

In this section we further relax the constraints of the problem and consider proper contact representations of graphs where vertices are represented by cuboids. We present two new (simple) proofs of the following result that was first established by Thomassen [22].

Theorem 6. Any planar graph has a proper contact representation by cuboids.

The idea behind the first proof is to use the concept of canonical ordering of a maximal planar graph, introduced by de Fraysseix, Pach, and Pollack in [14]. First, dummy edges are added to the input graph $G$ so that $G$ is a maximal planar graph. We draw the cuboids one at a time in the canonical order. We maintain an $(x, y)$-"staircase" profile of the active vertices of $G_{i}$ (that are the vertices on the external face of $G_{i}$ ). The cuboids representing active vertices of $G_{i}$ have their top sides lying on the plane $z=i$. The neighbors in $G_{i}$ of vertex $v_{i+1}$ are a consecutive sequence $v_{l}, v_{l+1}, \ldots, v_{r}$ of the active vertices of $G_{i}$. When the cuboid representing vertex $v_{i+1}$ is drawn, each cuboid representing a vertex $v_{j}$ such that $j \in\{l, l+1, \ldots, r\}$ and such that $\left(v_{j}, v_{i+1}\right)$ belongs to the input graph is slightly extended in the positive $z$-direction. The new vertex $v_{i+1}$ is represented by a cuboid placed on top of the cuboids representing its neighbors in $v_{l+1}, v_{l+2}, \ldots, v_{r-1}$. The cuboid representing $v_{i+1}$ touches each neighbor in $v_{l+1}, v_{l+2}, \ldots, v_{r-1}$ along a face parallel to the $(y, z)$-plane, and it touches $v_{l}$ and $v_{r}$ along faces parallel to the $(x, y)$-plane and to the $(x, z)$-plane. The active vertices of $G_{i+1}$ are extended in the positive $z$-direction so that their top sides lie on the plane $z=i+1$ and the invariant is maintained. See Fig.6. 


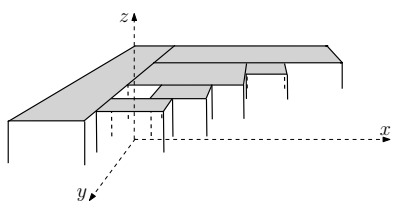

(a)

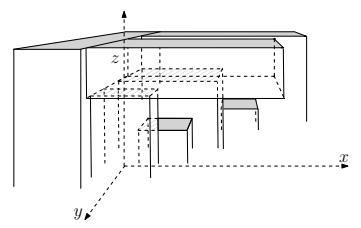

(b)

Fig. 6. Representation of $G_{i}$ (a) and representation of $G_{i+1}$ (b) by touching cuboids

The second proof uses Schnyder's realizers [21] to construct a proper contact representation of the input graph $G$ by cuboids. First, dummy edges are added to $G$ so that $G$ is a maximal planar graph. Later, contacts representing dummy edges in $G$ will be avoided by slightly shrinking the sides of some cuboids. Let $a_{1}, a_{2}$, and $a_{3}$ be the vertices incident to the external face of a planar embedding of $G$. A Schnyder realizer [21] of $G$ is a partition of the internal edges of $G$ into three sets $T_{1}, T_{2}$, and $T_{3}$ such that (1) for $i=1,2,3, T_{i}$ is a tree whose vertex set consists of the internal vertices of $G$ plus $a_{i}$, and (2) assuming that the edges of $T_{i}$ are directed towards $a_{i}$, for $i=1,2,3$, then the counterclockwise order of the edges around each internal vertex $v$ of $G$ consists of the edges: Leaving $v$ in $T_{1}$, then entering $v$ in $T_{3}$, then leaving $v$ in $T_{2}$, then entering $v$ in $T_{1}$, then leaving $v$ in $T_{3}$, and finally entering $v$ in $T_{2}$. We further add edges $\left(a_{2}, a_{1}\right)$, $\left(a_{3}, a_{2}\right)$, and $\left(a_{1}, a_{3}\right)$ to $T_{1}, T_{2}$, and $T_{3}$, respectively; see Fig. 7 .

Let $<_{X},<_{Y}$, and $<_{Z}$ be three total orders on the vertices of $G$ such that $<_{X}$ is consistent with $T_{1}, T_{2}^{-1}$, and $T_{3}^{-1}$, such that $<_{Y}$ is consistent with $T_{1}^{-1}, T_{2}$, and $T_{3}^{-1}$, and such that $<_{Z}$ is consistent with $T_{1}^{-1}, T_{2}^{-1}$, and $T_{3}$. Here, partial order $T_{i}^{-1}$ is the inverse of partial order $T_{i}$, for $i=1,2,3$. Orders $<_{X},<_{Y}$, and $<_{Z}$ exist and define a 3-dimensional representation of $G$ [20].

We use these three total orders to define a proper contact representation of $G$ by cuboids. For each vertex $v$ of $G$, the cuboid representing $v$, called the $v$-cuboid, is the region $[\mathbf{x}(v), \mathbf{X}(v)] \times[\mathrm{y}(v), \mathbf{Y}(v)] \times[\mathbf{z}(v), \mathbf{Z}(v)]$ defined as follows. Let $\mathbf{X}(v), \mathbf{Y}(v)$, and $\mathrm{Z}(v)$ be the rank of $v$ in the inverse of $<_{X},<_{Y}$, and $<_{Z}$, respectively. (Using the inverse of $<_{X},<_{Y}$, and $<_{Z}$ yields to contact representations similar to the T-contact systems as constructed in [13].) For each edge $(u, v) \in T_{1}$, let $\times(u)=\mathrm{X}(v)$. Let $\mathrm{x}\left(a_{1}\right)=\mathrm{x}\left(a_{3}\right)=0$. Since every vertex internal vertex of $G$, as well as vertex $a_{2}$, has outdegree one in $T_{1}$, this defines $\times(u)$ for all the vertices in $G$. If $(u, v)$ is a dummy edge, then add $\epsilon$ to $\times(u)$, where $0<\epsilon<1$. For each edge $(u, v) \in T_{2}$, let $\mathrm{y}(u)=\mathrm{Y}(v)$ (let $\mathrm{y}(u)=\mathrm{Y}(v)+\epsilon$ if $(u, v)$ is a dummy edge); further, let $\mathrm{y}\left(a_{1}\right)=\mathrm{y}\left(a_{2}\right)=0$. For each edge $(u, v) \in T_{3}$, let $\mathrm{z}(u)=\mathrm{Z}(v)$ (let $\mathrm{z}(u)=\mathrm{Z}(v)+\epsilon$ if $(u, v)$ is a dummy edge); further, let $\mathrm{z}\left(a_{2}\right)=\mathrm{z}\left(a_{3}\right)=0$. Let $B\left(<_{X},<_{Y},<_{Z}\right)$ be the set of $v$-cuboids defined by $<_{X},<_{Y}$, and $<_{Z}$ (obtained from the Schnyder realizer $\left(T_{1}, T_{2}, T_{3}\right)$ of $G$ ); see Fig. 7 .

Lemma 3. $B\left(<_{X},<_{Y},<_{Z}\right)$ is a proper contact representation of $G$ by cuboids.

Proof: By construction the $a_{1}$-cuboid and the $a_{2}$-cuboid touch along a face parallel to the $(y, z)$-plane, unless edge $\left(a_{2}, a_{1}\right)$ is dummy, in which case $\times\left(a_{2}\right)=\mathrm{X}\left(a_{1}\right)+\epsilon$, thus such cuboids are separated by a plane parallel to the $(y, z)$-plane and do not touch. Analogously, edges $\left(a_{3}, a_{2}\right)$ and $\left(a_{1}, a_{3}\right)$ are correctly represented in the drawing. 

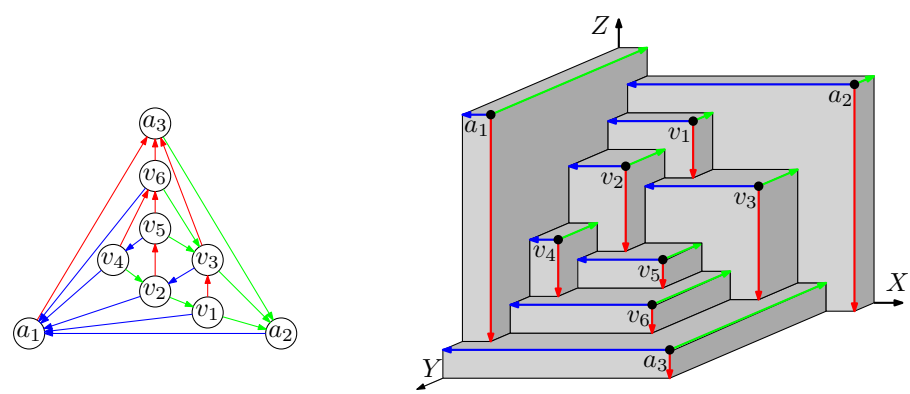

Fig. 7. A Schnyder realizer with $<_{X}: a_{2} a_{3} v_{3} v_{6} v_{5} v_{1} v_{2} v_{4} a_{1},<_{Y}: a_{3} a_{1} v_{6} v_{4} v_{5} v_{2} v_{3} v_{1} a_{2}$, and $<_{Z}: a_{1} a_{2} v_{1} v_{2} v_{3} v_{4} v_{5} v_{6} a_{3}$ and the corresponding proper contact representation by cuboids.

Consider an internal directed edge $(u, v)$ in $G$ and assume it belongs to $T_{3}$. The argument is similar if it belongs to $T_{1}$ or to $T_{2}$. If $(u, v)$ is dummy, then $\mathrm{z}(u)=\mathrm{Z}(v)+$ $\epsilon$, thus the $u$-cuboid and the $v$-cuboid are separated by a plane parallel to the $(x, y)$ plane and do not touch in the constructed representation. Otherwise, we show that the corner $(\mathrm{X}(u), \mathrm{Y}(u), \mathrm{z}(u))$ of the $u$-cuboid lies in the interior of the face $([\mathrm{x}(v), \mathrm{X}(v)]$, $[\mathrm{y}(v), \mathrm{Y}(v)], \mathrm{Z}(v))$ of the $v$-cuboid. By construction, $\mathrm{z}(u)=\mathrm{Z}(v)$. Since $(u, v) \in T_{3}$, $v<_{X} u$ and $v<_{Y} u$ thus $\mathrm{X}(u)<\mathrm{X}(v)$ and $\mathrm{Y}(u)<\mathrm{Y}(v)$. If $v$ is an external vertex then $v=a_{3}$ so $\mathrm{X}(u)>\mathrm{x}(v)=0$ and $\mathrm{Y}(u)>\mathrm{y}(v)=1$. Otherwise, to establish $\mathrm{X}(u)>\mathrm{x}(v)$, let $(v, w)$ be the edge leaving $v$ in $T_{1}$. Let $u=u_{0}, u_{1}, \ldots, u_{k}=w$ be the neighbors of $v$ in clockwise order from $u$ to $w$. By Property (2) of Schnyder realizers, the edges $\left(u_{i}, v\right)$ are entering $v$ in $T_{3}$, for $i=0,1, \ldots k-1$. Since, for all $i=0,1, \ldots, k-1$, the three vertices $u_{i}, u_{i+1}, v$ form a cycle, by Property (2) of Schnyder realizers $\left\{u_{i}, u_{i+1}\right\}$ is either entering $u_{i+1}$ in $T_{1}$ or leaving $u_{i+1}$ in $T_{2}$. In either case, $u_{i}<_{X} u_{i+1}$, thus, by transitivity, $\mathbf{X}(u)>\mathbf{X}(w)=\mathrm{x}(v)$. A similar argument can be used to show that $\mathrm{Y}(u)>\mathrm{y}(v)$. Hence, the $u$-cuboid and the $v$-cuboid touch along a face parallel to the $(x, y)$-plane, thus concluding the proof.

\section{Open Problems}

Open questions about representating graphs as contact graphs of cuboids in 3D include:

1. Does every planar graph admit a proper contact representation by cubes?

2. Is it possible to characterize/recognize the class of graphs that have a proper contact representation by cubes (e.g., observe that $K_{5}$ is not representable while $K_{3,3}$ is)?

3. Is it possible to characterize/recognize the class of planar graphs that have a proper contact representation by unit cubes? Similarly for binary trees?

Acknowledgments. This research, with NSERC support, began at the McGill/INRIA/ University of Victoria 2012 Workshop at Bellairs Research Institute. The authors thank the other participants. 


\section{References}

1. Alam, M.J., Biedl, T.C., Felsner, S., Kaufmann, M., Kobourov, S.G., Ueckerdt, T.: Computing cartograms with optimal complexity. In: Dey, T.K., Whitesides, S. (eds.) Symposium on Computational Geometry, SoCG 2012, pp. 21-30 (2012)

2. Badent, M., Binucci, C., Giacomo, E.D., Didimo, W., Felsner, S., Giordano, F., Kratochvíl, J., Palladino, P., Patrignani, M., Trotta, F.: Homothetic triangle contact representations of planar graphs. In: Bose, P. (ed.) Canadian Conference on Computational Geometry, CCCG 2007, pp. 233-236 (2007)

3. Battista, G.D., Eades, P., Tamassia, R., Tollis, I.G.: Graph Drawing: Algorithms for the Visualization of Graphs. Prentice-Hall (1999)

4. Borchard-Ott, W.: Crystallography. Springer (2011)

5. Breu, H.: Algorithmic Aspects of Constrained Unit Disk Graphs. Ph.D. thesis, The University of British Columbia, Canada (1996)

6. Buchsbaum, A.L., Gansner, E.R., Procopiuc, C.M., Venkatasubramanian, S.: Rectangular layouts and contact graphs. ACM Transactions on Algorithms 4(1) (2008)

7. Czyzowicz, J., Kranakis, E., Krizanc, D., Urrutia, J.: Discrete realizations of contact and intersection graphs. International Journal of Pure and Applied Mathematics 13(4), 429-442 (2004)

8. de Fraysseix, H., Ossona de Mendez, P.: Representations of Planar Graphs by Segments. Colloquia Mathematica Societatis János Bolyai, vol. 63, pp. 109-117. North-Holland (2007)

9. Duncan, C.A., Gansner, E.R., Hu, Y.F., Kaufmann, M., Kobourov, S.G.: Optimal polygonal representation of planar graphs. Algorithmica 63(3), 672-691 (2012)

10. Eades, P., Whitesides, S.: The logic engine and the realization problem for nearest neighbor graphs. Theoretical Computer Science 169(1), 23-37 (1996)

11. Felsner, S.: Rectangle and square representations of planar graphs. In: Pach, J. (ed.) Thirty Essays on Geometric Graph Theory. Springer (2012)

12. Felsner, S., Francis, M.C.: Contact representations of planar graphs with cubes. In: Hurtado, F., van Kreveld, M.J. (eds.) Symposium on Computational Geometry, SoCG 2011, pp. 315320 (2011)

13. de Fraysseix, H., Ossona de Mendez, P., Rosenstiehl, P.: On triangle contact graphs. Combinatorics, Probability \& Computing 3, 233-246 (1994)

14. de Fraysseix, H., Pach, J., Pollack, R.: How to draw a planar graph on a grid. Combinatorica 10(1), 41-51 (1990)

15. He, X.: On floor-plan of plane graphs. SIAM Journal on Computing 28(6), 2150-2167 (1999)

16. Kaufmann, M., Wagner, D. (eds.): Drawing graphs: methods and models. Springer (2001)

17. Koebe, P.: Kontaktprobleme der konformen Abbildung. Berichte über die Verhandlungen der Sächsischen Akad. der Wissenschaften zu Leipzig. Math.-Phys. Klasse 88, 141-164 (1936)

18. Koźmiński, K., Kinnen, E.: Rectangular duals of planar graphs. Networks 15, 145-157 (1985)

19. Leinwand, S.M., Lai, Y.T.: An algorithm for building rectangular floor-plans. In: Lambert, P.H., Ofek, H., O'Neill, L.A., Pistilli, P.O., Losleben, P., Nash, J.D., Shaklee, D.W., Preas, B.T., Lerman, H.N. (eds.) Design Automation Conference, DAC 1984, pp. 663-664 (1984)

20. Schnyder, W.: Planar graphs and poset dimension. Order 5(4), 323-343 (1989)

21. Schnyder, W.: Embedding planar graphs on the grid. In: Symposium on Discrete Algorithms, SODA 1990, pp. 138-148 (1990)

22. Thomassen, C.: Interval representations of planar graphs. Journal of Combinatorial Theory, Series B 40(1), 9-20 (1986)

23. Ungar, P.: On diagrams representing graphs. Journal of the London Mathematical Society 28, 336-342 (1953) 